\title{
The Primary Spontaneous Pneumothorax trial: A critical appraisal from the surgeon's perspective
}

\author{
Andrew E. Giles, MD, MPH, ${ }^{a}$ Biniam Kidane, MD, MSc, ${ }^{\text {a,b,c }}$ Morgan Schellenberg, MD, MPH, ${ }^{\mathrm{d}}$ and \\ Chad G. Ball, MD, MSc, ${ }^{e}$ on behalf of the Evidence Based Reviews in Surgery (EBRS) Group*
}

This paper is meant to provide a synopsis of a systematic critical evaluation of a high-impact randomized trial. To accomplish this, headings have been applied to provide structure to the critique process, but the text is mainly narrative to improve readability. It is derived from the methods used in the Evidence Based Reviews in Surgery (EBRS) program and is intended to provide the readership with an approachable review while simultaneously illustrating one method of critically evaluating published literature. The key questions and issues we feel should be addressed are framed by these headers, and summary sentences are provided in italics.

\section{STATE OF THE EVIDENCE}

\section{Rationale for the Study}

The primary spontaneous pneumothorax (PSP) is an enigmatic condition. Relatively little attention has been dedicated to studying its pathophysiology and management in a systematic manner. Over the decades, management has largely consisted of pleural drainage, with observation reserved for small and asymptomatic cases. This is reflected in the existing guidelines, ${ }^{1-5}$ with the American College of Chest Physicians recommendations ${ }^{1}$ dating 2001, and the most recent from the European Respiratory Society ${ }^{4}$ in 2015 . Table 1 provides a summary of guideline recommendations. There are no randomized trials comparing observation to intervention, ${ }^{6}$ until now.

\section{Clinical Question}

The historical precedent for treating PSP without intervention originates with a cohort study ${ }^{7}$ from the United Kingdom in 1966, in which local policy dictated that all uncomplicated pneumothoraces were initially treated with observation alone. This was a successful strategy for $83 \%$ of patients with PSP.

From the a Section of Thoracic Surgery, Department of Surgery, Max Rady College of Medicine, and ${ }^{\mathrm{b}}$ Department of Community Health Sciences, University of Manitoba, Winnipeg, Manitoba, Canada; ${ }^{\mathrm{c}}$ Research Institute in Oncology \& Hematology, Cancer Care Manitoba, Winnipeg, Manitoba, Canada; ${ }^{\mathrm{d} D i v i s i o n}$ of Acute Care Surgery, LAC + USC Medical Center, Los Angeles, Calif; and ${ }^{\mathrm{e}}$ Department of Surgery, University of Calgary, Calgary, Alberta, Canada.

Drs Giles and Kidane contributed equally to this article.

* See Members of EBRS Group at end of manuscript.

Received for publication Jan 1, 2021; revisions received Feb 7, 2021; accepted for publication Feb 12, 2021; available ahead of print Feb 24, 2021

Address for reprints: Biniam Kidane, MD, MSc, Health Sciences Centre, GE-611, 820 Sherbrook St, Winnipeg, Manitoba, Canada, R3A 1R9 (E-mail: bkidane@ hsc.mb.ca).

J Thorac Cardiovasc Surg 2021;162:1428-32

$0022-5223 / \$ 36.00$

Copyright (c) 2021 by The American Association for Thoracic Surgery

https://doi.org/10.1016/j.jtcvs.2021.02.070

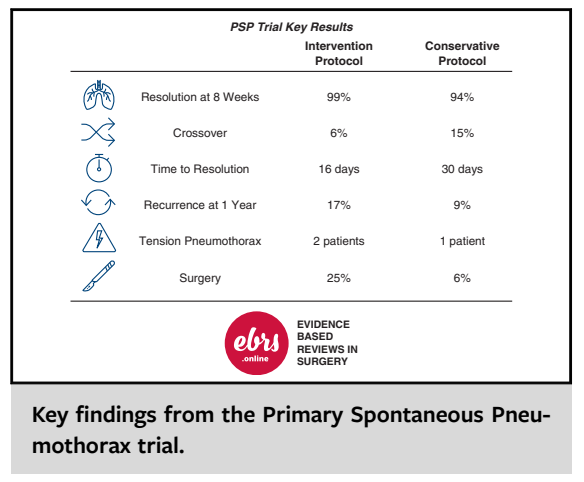

CENTRAL MESSAGE

Expectant management of moderate and large primary spontaneous pneumothorax may be reasonable with appropriate patient counseling, recognizing that a sizeable proportion still require intervention.

See Commentaries on pages 1433 and 1434 .

These patients were encouraged to work and resume normal life. Over a span of 5 years, recurrence occurred in only $11 \%$ of patients. A more recent observational study from $2008^{8}$ confirmed that $45 \%$ of patients with PSP were able to be managed with outpatient observation; however, only $5 \%$ of patients in this group had large pneumothoraces.

\section{Is There Clinical Equipoise?}

Spurred on by these studies and their own observations, Brown and colleagues ${ }^{9}$ conducted a retrospective review, finding that $33 \%$ of patients with PSP $(20 \%$ of these were moderate in size) were managed without intervention and that recurrence occurred less frequently in this group $(5 \%)$ versus those who had pleural drainage $(17 \%)$. Armed with these findings suggesting clinical equipoise, they went on to conduct the PSP trial ${ }^{10}$ to formally address whether observation is safe and similarly effective in managing moderate and large primary spontaneous pneumothorax.

\section{Purpose of This Review}

This study was authored primarily by emergency medicine and pulmonary medicine physicians and published in the New 
TABLE 1. Summary of existing guidelines

\begin{tabular}{|c|c|c|c|c|}
\hline Guidelines & $\begin{array}{c}\text { Year } \\
\text { published }\end{array}$ & $\begin{array}{l}\text { Definition of large } \\
\text { pneumothorax }\end{array}$ & $\begin{array}{l}\text { Management-small } \\
\text { pneumothorax }\end{array}$ & $\begin{array}{c}\text { Management-large } \\
\text { pneumothorax }\end{array}$ \\
\hline $\begin{array}{l}\text { American College of } \\
\text { Chest Physicians }{ }^{1}\end{array}$ & 2001 & $>3 \mathrm{~cm}$ & $\begin{array}{l}\text { ED observation and outpatient } \\
\text { follow-up }\end{array}$ & Catheter insertion \\
\hline $\begin{array}{l}\text { Therapeutic Guidelines } \\
\text { (Australia) })^{5}\end{array}$ & 2005 & $\begin{array}{l}>2 \mathrm{~cm} \text { from lateral } \\
\text { chest wall }\end{array}$ & Observation at home or in hospital & $\begin{array}{l}\text { Asymptomatic: observation } \\
\text { Symptomatic: aspirate }\end{array}$ \\
\hline $\begin{array}{l}\text { Belgian Society of } \\
\text { Pneumology }\end{array}$ & 2006 & $\begin{array}{l}\text { Lung dehiscence } \\
\text { entire lateral chest } \\
\text { wall }\end{array}$ & $\begin{array}{l}\text { ED observation and outpatient } \\
\text { follow-up }\end{array}$ & $\begin{array}{l}\text { Aspirate or catheter } \\
\text { Catheter if aspiration } \\
\text { unsuccessful }\end{array}$ \\
\hline British Thoracic Society ${ }^{2}$ & 2010 & $>2 \mathrm{~cm}$ & $\begin{array}{l}\text { ED observation and outpatient } \\
\text { follow-up }\end{array}$ & $\begin{array}{l}\text { Aspirate } \\
\text { Catheter if unsuccessful }\end{array}$ \\
\hline $\begin{array}{l}\text { European Respiratory } \\
\text { Society task force } \\
\text { statement }^{4}\end{array}$ & 2015 & $\begin{array}{l}\text { Not given; treat based } \\
\text { on symptoms }\end{array}$ & $\begin{array}{l}\text { Minimal or no symptoms: } \\
\text { observation } \\
\text { Symptomatic: aspiration or } \\
\text { small-bore catheter }\end{array}$ & \\
\hline
\end{tabular}

$E D$, Emergency department.

England Journal of Medicine. However, its impact will extend beyond these specialties. Surgeons are routinely involved in the management of patients with pneumothorax, particularly those who do not respond to observation and simple pleural drainage procedures. It was therefore selected for review as part of the Evidence Based Reviews in Surgery (https://ebrs. online). This paper serves as a synopsis of the appraisals by the expert clinical and methodologic surgeon reviewer panel. It has been structured to emphasize a systematic approach to evaluating a randomized trial.

\section{STUDY DESIGN AND METHODOLOGY \\ Trial Design}

Brown and colleagues ${ }^{9}$ conducted a multicenter prospective randomized control trial at 39 medical facilities in Australia and New Zealand. They enrolled patients with moderate and large primary spontaneous pneumothorax aged 14 to 50 years old. The age criterion was to decrease the likelihood of secondary spontaneous pneumothorax and because previous observational studies have shown that older patients are less likely to have successful resolution without chest drainage. Key exclusion criteria were small size $(<32 \%$ by the Collins method), bilateral or previous pneumothoraces, and anticipated travel. Patients presenting with tension physiology were also excluded. Overall, this study investigated a carefully selected group of patients with moderate and large primary spontaneous pneumothorax.

\section{Blinding and Power Calculations}

There was no blinding due to the nature of the intervention, although a secondary outcome was radiograph review by blinded radiologists. It was designed as a noninferiority trial, with an absolute noninferiority margin of $9 \%$. This allows for up to a $9 \%$ greater radiographic nonresolution rate at 8 weeks for the conservative management arm. The rationale, as explained in the study protocol, is as follows. A $99 \%$ resolution rate is anticipated for traditional interventional management. A 90\% resolution rate (ie, 1 in 10 chance of failure) would be acceptable if it was offset by avoidance of chest tube insertion and associated issues. It is unclear if patients were involved in deciding on choice and magnitude of these trade-offs. There was no blinding, and to be noninferior, the conservative management strategy had to achieve a $90 \%$ radiographic resolution at 8 weeks, compared with anticipated $99 \%$ for the interventional strategy.

\section{Management Protocols}

The conservative management arm consisted of iterative assessments in the emergency department (ED) and a repeat chest radiograph (CXR) in 4 hours. If patients exhibited normal vital signs, were ambulatory, and not requiring oxygen, they were discharged with telephone follow-up in 24 to 72 hours and outpatient CXRs every 2 weeks until resolution. Patients in the intervention arm underwent insertion of a small bore ( $12 \mathrm{~F}$ or smaller) drain attached to water seal. After 1 hour, CXR was repeated; if pneumothorax was small and there was no air leak, it was clamped. CXR was repeated 4 hours later. If there was no reaccumulation and the patient met the same discharge criteria, the drain was removed and they were discharged home. Otherwise, the drain was reopened to water seal and they were admitted to hospital for further management. The follow-up schedule mirrored that of the conservative group. Both groups were serially monitored in the ED, with discharge or admission based on clinical improvement.

\section{Primary Outcome}

The primary outcome was full lung re-expansion as assessed on radiograph within 8 weeks. The original analysis plan was to use logistic regression for the primary outcome, and did not specify how missing data would be dealt with. The main analysis excluded missing data points. Two secondary analyses were conducted: (1) Patients with CXRs performed up to 9 weeks were included; (2) All missing data was considered as treatment failure. The primary outcome was radiographic resolution at 8 weeks; sensitivity analyses were performed based on deviation from this.

\section{Secondary Outcomes}

Secondary outcomes included a per-protocol analysis of the primary outcome, adverse events, resolution as assessed by blinded radiologists, pneumothorax reoccurrence, time until symptom resolution, patient satisfaction, duration of hospital admission, number of procedures and investigations, and days off work.

\section{SUMMARY OF RESULTS \\ Results: Primary Outcome}

Brown and colleagues ${ }^{9}$ screened 2637 patients and randomized 316, of whom $154(49 \%)$ were randomized to the intervention arm and $162(51 \%)$ to the conservative management arm. In the primary analysis, radiographic resolution at 8 weeks was noninferior after conservative management as compared to intervention (94\% vs $99 \%$ resolution; Figure 1). However, in the more conservative iteration of the analysis with missing data patients considered to have not achieved the primary outcome, the risk difference between groups was $-11.0 \%$, violating the noninferiority margin. Table 2 includes a summary of the primary and secondary 


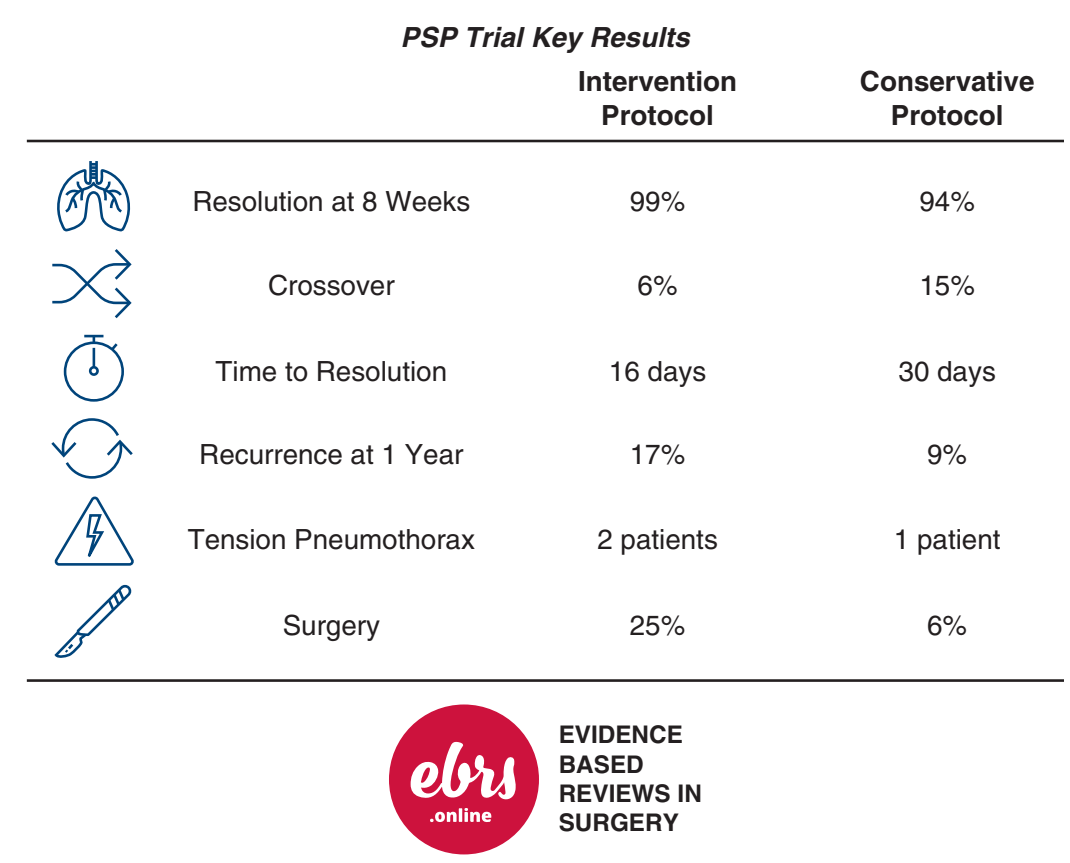

FIGURE 1. Major findings from the Primary Spontaneous Pneumothorax trial. ${ }^{10}$

analyses. Noninferiority was met in the primary analysis but failed under conservative assumptions.

\section{Results: Crossover and Secondary Outcomes}

Ten patients $(6.5 \%)$ assigned to the intervention group declined intervention. Twenty-five patients (15.4\%) assigned to the conservative management group underwent intervention, most for physiologic derangements or intolerable symptoms.

Among secondary outcomes, time to pneumothorax resolution was shorter in the intervention group (16 vs 30 days; hazard ratio, 0.49 ; 95\% confidence interval [CI], 0.39$0.63)$. Time to symptom resolution and satisfaction were no different between groups. Recurrence in the first year was more common after intervention than conservative management $(16.8 \%$ vs $8.8 \%$; absolute risk difference, $8.0 \%$; $95 \%$ CI, 0.5-15.4). Hospital length of stay and duration of time off from work were also longer after intervention. Adverse events were more common in the intervention group (relative risk, 3.32; 95\% CI, 1.85-5.95), although these were largely drain-related issues (eg, retained suture). Tension pneumothorax occurred in 2 patients in the intervention group and 1 in the conservative management group. Crossover was greater in the conservative protocol group, long-term resolution was reduced. Time to radiographic resolution was shorter with intervention, adverse events were higher.

\section{EBRS APPRAISAL}

Risk of bias considerations take into account creation and maintenance of prognostic balance and the ability to administer both interventions with similar expertise.

\section{Were Patients Similar at the Start of the Study?}

Randomization and allocation concealment were robust, accomplished through real-time web-based randomization.
Aside from a slightly longer duration of symptoms and a greater proportion of smokers among those assigned to the intervention arm, the groups were otherwise similar at baseline. Blinding was not possible due to the nature of the treatment arms. Attempts to minimize bias included the use of an objective outcome measure (radiographic resolution) and blinded radiologist assessment as a secondary outcome. Radiologists were more likely to report radiographs as nonresolved $(-5.9 \%, P<.001)$, but noninferiority of conservative management was maintained. Randomization and concealment of allocation were appropriate; blinding was not possible but mitigation efforts were made.

\section{Were Patients Treated Similarly and Accounted for by Completion of the Study?}

Completeness of follow-up at the end of the study was good, with only 5 patients in the intervention group and 3 in the conservative management group lost to follow-up. However, the main analysis specified radiographic assessment within 8 weeks, which resulted in 7 patients in the intervention group and 16 in the conservative group being excluded. While the primary end-point of noninferiority was met, under conservative assumptions (imputing all missing data points as nonresolution) the conservative management group no longer met noninferiority criteria. The authors should be commended for their use of appropriate sensitivity analyses with varying (and conservative) loss to follow-up assumptions. ${ }^{11}$

Outcomes were analyzed in an intent-to-treat fashion, preserving allocation. Per-protocol analysis is crucial to report in noninferiority trials, and noninferiority was preserved with this analysis despite the unequal crossover between groups $(6 \%$ intervention to conservative; $15 \%$ in the opposite direction). 
TABLE 2. Results of the main, sensitivity, and per-protocol analyses of the primary outcome, radiographic resolution of pneumothorax at 8 weeks ${ }^{10}$

\begin{tabular}{llcccc}
\hline & \multicolumn{4}{c}{ Resolution } & \multicolumn{3}{c}{} \\
\cline { 2 - 5 } & Treatment of missing data & Intervention strategy & Conservative strategy & Risk difference $(\mathbf{9 5} \%$ CI) & $P$ value \\
\hline Primary Outcome & $>56 \mathrm{~d}=$ missing & $129 / 131(98.5 \%)$ & $118 / 125(94.4 \%)$ & $-4.1 \%(-8.6$ to 0.5$)$ & .02 \\
Sensitivity analysis 1 & $>63 \mathrm{~d}=$ missing & $134 / 136(98.5 \%)$ & $129 / 136(94.9 \%)$ & $-3.7 \%(-7.9$ to 0.6$)$ & - \\
Sensitivity analysis 2 & $>56 \mathrm{~d}=$ failure & $129 / 138(93.5 \%)$ & $118 / 143(82.5 \%)$ & $-11.0 \%(-18.4$ to -3.5$)$ & - \\
Per-protocol & $>56 \mathrm{~d}=$ missing & $124 / 126(98.4 \%)$ & $123 / 130(94.6 \%)$ & $-3.8 \%(-8.3$ to 0.7$)$ & - \\
\hline
\end{tabular}

While randomization confers prognostic balance, it is maintained through blinding and equal application of cointerventions. Because blinding was not possible, the latter is crucial and merits consideration. Threats to expertise occur in 2 main areas: potential bias in application of the clinical criteria and the low number of patients treated at each site. Regarding the former, treating clinicians may consider patient symptoms to be less significant with a drainage tube in situ because they are reassured about the safety of that strategy; conversely, they may falsely attribute symptoms to the pneumothorax that actually are secondary to an intercostal catheter. The direction and magnitude of such hypothetical effects is hard to determine. Concerning enrollment volumes, with very few patients enrolled at each site it would be challenging to develop experience and procedural familiarity. This could lead to variability in individual patient management between management protocols and sites.

There were issues with follow-up within the primary outcome period, but sensitivity analyses were appropriate. Lack of blinding and possible unequal application of treatments increase risk of bias, but not in a clear uniform direction.

\section{WHAT CAN WE TAKE FROM THIS STUDY?}

In a cohort of 316 highly selected patients with moderateto-large PSP, a conservative management strategy fell within the $9 \%$ noninferiority margin of intervention and was welltolerated. While this was not robust to conservative statistical assumptions, the authors ensured this was clearly stated and explored appropriately. Recurrence at 1 year was lower in the conservative management group, which lends credence to the notion that PSP is a relatively benign process and that gradual re-expansion allows for better healing of the inciting lung defect. ${ }^{12}$ Adverse events overall were greater in the intervention group but mostly related to the drain itself. Among patients in the conservative management group who required intervention, adverse events related to the drain were more common. This may be related to the need for pleural drainage in the event of clinical decompensation, with tube placement being done under more strained circumstances. Serious complications such as tension pneumothorax occurred in both groups, alarmingly 2 of them following discharge.

\section{Can the Results of This Study be Applied to My Patients?}

While the message of this study could easily be interpreted as "no intervention is just as good as (if not preferable to) intervention," it is crucial to be clear about the conduct of the study. The authors compared management strategies, which could be thought of as upfront intervention versus observation with/without intervention, rather than a strict comparison of providing or withholding pleural drainage. One cannot extrapolate this study to all-comers in the ED. Patients cannot have underlying lung pathology, must be reliable and able to attend frequent follow-up, and must match the strict inclusion criteria. They should be advised that resolution will take longer without intervention, although they may not experience related symptoms. Patients must be carefully counseled on the $1 \%$ risk of tension pneumothorax and warned that this may occur outside of hospital. The attending clinicians must be flexible in their approach and prepared to intervene on the $15 \%$ of patients who will require pleural drainage due to clinical deterioration or persistent symptoms. Patients will still require surgery, as did 49 patients $(16 \%)$ enrolled in this study.

As such, surgeons will still be called upon to assist in management of these patients. It is necessary to understand the paradigm shift that this study affirms, and that management strategies will evolve as these findings are disseminated and incorporated into outdated guidelines. This study is the first to bring rigor to the legitimacy of a primarily noninterventional strategy for PSP, and other trials are likely to follow. As managers of the end point of this condition, surgeons would do well to appreciate the lessons in lung healing physiology that are suggested here. Involvement of surgeons in future studies is necessary to provide the perspective beyond the ED that we are familiar with.

\section{Were All Patient-Important Outcomes Considered?}

There are other peculiarities to take into consideration for a complete understanding of this landmark trial. First, choice of the primary outcome. Radiographic resolution is an objective measure and allows for a tidy comparative analysis. However, this may not be considered a patientimportant outcome. If the goal is to determine meaningful resolution of pneumothorax, functional assessment would be a more appropriate outcome, ${ }^{13,14}$ given that PSP primarily affects young, otherwise active, and economically engaged individuals. Furthermore, choice of resolution within 8 weeks is derived from the 1966 observational study, ${ }^{7}$ as most pneumothoraces had resolved radiologically within this time frame. For this trial, patients were not consulted to determine whether they were willing to wait up to 8 weeks for their pneumothorax to resolve, or what is an 
acceptable noninferiority margin. Although satisfaction scores were comparable between management strategies, some patients will be unwilling to accept the uncertainty and longer time to resolution with nonintervention.

\section{Were Enrolled Patients Administered Similar Treatment?}

The 316 enrolled patients were divided among 39 different treatment sites, or approximately 8 patients per hospital. Presumably the treating clinicians at each health facility caring for pneumothorax were already using both management strategies. However, within the confines of the study this does call into question uncertainty regarding expertise and consistency of protocol administration at each site. Given that only 4 pleural drains were placed per site, and most adverse events were related to drain management, there arises uncertainty about the ability to administer consistent care across study sites with so few interventions each. This may be conceptually extended to the decreased skill with insertion of and familiarity managing pleural drainage catheters if the non-interventional protocol gains traction, leading to greater rates of complications among the subset of patients that inevitably require intervention.

\section{Are the Likely Treatment Benefits Worth the Potential Harms and Costs?}

The current standard of care for moderate and large symptomatic pneumothorax is drainage; thus, it is incumbent on trialists to establish the safety of a novel management approach. In particular, tension pneumothorax is poorly understood, with some experts describing it as extremely rare and making "no physiologic sense" $"$; meanwhile, a $12 \%$ incidence has also been documented, ${ }^{15}$ although bearing no statistical association with hospital mortality. This discrepancy is largely related to detection method, but even with stringent physiologic criteria of hypotension, tension occurred in $1 \%$ of the patients in the PSP trial. A large trial powered to examine composite end points of serious adverse events, tension pneumothorax, and death remains to be completed before conservative management can be unreservedly advocated.

\section{FINAL THOUGHTS}

\section{Does the Evidence Support the Conclusion?}

The PSP trial is a major step forward in extending a conservative management strategy, which has long been used for small spontaneous primary pneumothoraces, to treatment of moderate and large PSP. Long-term recurrence may be lower without pleural intervention. While noninferiority was achieved in the primary analysis, this was subject to statistical frailty. Pleural drainage was still necessary in $15 \%$ of patients under the conservative management strategy, and among both groups, surgery was eventually required in $16 \%$. Overall, this appears to be a safe approach, but only when the treatment strategies are well understood, are properly applied to a specific set of patients, and patients have been fully informed.

\section{Conflict of Interest Statement}

The authors reported no conflicts of interest.

The Journal policy requires editors and reviewers to disclose conflicts of interest and to decline handling or reviewing manuscripts for which they may have a conflict of interest. The editors and reviewers of this article have no conflicts of interest.

Members of Evidence Based Reviews in Surgery (EBRS) Group: Carl Brown, Elijah Dixon, Neel Datta, Jason Park, Fahima Dossa, Ameer Farooq, Nancy Baxter, Mantaj Brar, Prosanto Chaudhury, Sandra deMontburn, Justin Dimick, Bill Fitzgerald, Samantha Hendren, Lillian Kao, Andrew Kirkpatrick, Steve Latosinsky, Robin McLeod, Arden Morris, Tim Pawlik, Kjetil Söreide, Malin Sund, Des Winter, Bas Wjinhoven.

\section{References}

1. Baumann MH, Strange C, Heffner JE, Light R, Kirby T, Klein J, et al. Management of spontaneous pneumothorax: an American College of Chest Physicians Delphi consensus statement. Chest. 2001;119:590-602.

2. MacDuff A, Arnold A, Harvey J. Management of spontaneous pneumothorax: British Thoracic Society pleural disease guideline 2010. Thorax. 2010;65(suppl 2): $18-31$.

3. De Leyn P, Lismonde M, Ninane V, Noppen M, Slabbynck H, Van Meerhaeghe A, et al; Belgian Society of Pneumology. Guidelines on the management of spontaneous pneumothorax. Acta Chir Belg. 2005;105:265-7.

4. Tschopp J-M, Bintcliffe O, Astoul P, Canalis E, Driesen P, Janssen J, et al. ERS task force statement: diagnosis and treatment of primary spontaneous pneumothorax. Eur Respir J. 2015;46:321-35.

5. Kelly A-M, Clooney M, Spontaneous Pneumothorax Australia Study Group. Deviation from published guidelines in the management of primary spontaneous pneumothorax in Australia. Intern Med J. 2008;38:64-7.

6. Ashby M, Haug G, Mulcahy P, Ogden KJ, Jensen O, Walters JAE. Conservative versus interventional management for primary spontaneous pneumothorax in adults. Cochrane Database Syst Rev. 2014;2014:CD010565.

7. Stradling P, Poole G. Conservative management of spontaneous pneumothorax. Thorax. 1966;21:145-9.

8. Kelly A-M, Kerr D, Clooney M. Outcomes of emergency department patients treated for primary spontaneous pneumothorax. Chest. 2008;134:1033-6.

9. Brown SGA, Ball EL, Macdonald SPJ, Wright C, Taylor DMcD. Spontaneous pneumothorax; a multicentre retrospective analysis of emergency treatment, complications and outcomes. Intern Med J. 2014;44:450-7.

10. Brown SGA, Ball EL, Perrin K, Asha SE, Braithwaite I, Egerton-Warburton D, et al. Conservative versus interventional treatment for spontaneous pneumothorax. N Engl J Med. 2020;382:405-15.

11. Kidane B, Plourde M, Chadi SA, Iansavitchene A, Meade MO, Parry NG, et al. The effect of loss to follow-up on treatment of blunt traumatic thoracic aortic injury. J Vasc Surg. 2015;61:1624-34.

12. Simpson G. Spontaneous pneumothorax: time for some fresh air. Intern Med J. 2010;40:231-4.

13. Myles PS, Hunt JO, Nightingale CE, Fletcher H, Beh T, Tanil D, et al. Development and psychometric testing of a quality of recovery score after general anesthesia and surgery in adults. Anesth Analg. 1999;88:83-90.

14. Rahimi K, Malhotra A, Banning AP, Jenkinson C. Outcome selection and role of patient reported outcomes in contemporary cardiovascular trials: systematic review. BMJ. 2010;341:c5707.

15. Yoon JS, Choi SY, Suh JH, Jeong JY, Lee BY, Park YG, et al. Tension pneumothorax, is it a really life-threatening condition? J Cardiothorac Surg. 2013;8:197-203.

Key Words: pneumothorax, tension pneumothorax, emergency medicine, thoracic surgery 